\title{
Domain wall stability in ferroelectrics with space charges
}

Cite as: J. Appl. Phys. 115, 084110 (2014); https://doi.org/10.1063/1.4866359

Submitted: 22 November 2013 - Accepted: 08 February 2014 • Published Online: 27 February 2014

Yinan Zuo, Yuri A. Genenko, Andreas Klein, et al.
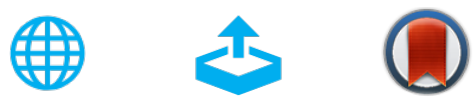

View Online

Export Citation

CrossMark

\section{ARTICLES YOU MAY BE INTERESTED IN}

Charge compensation of head-to-head and tail-to-tail domain walls in barium titanate and its influence on conductivity

Journal of Applied Physics 116, 044109 (2014); https://doi.org/10.1063/1.4891259

$\mathrm{BaTiO}_{3}$-based piezoelectrics: Fundamentals, current status, and perspectives

Applied Physics Reviews 4, 041305 (2017); https: / doi.org/10.1063/1.4990046

Ferroelectric thin films: Review of materials, properties, and applications

Journal of Applied Physics 100, 051606 (2006); https://doi.org/10.1063/1.2336999

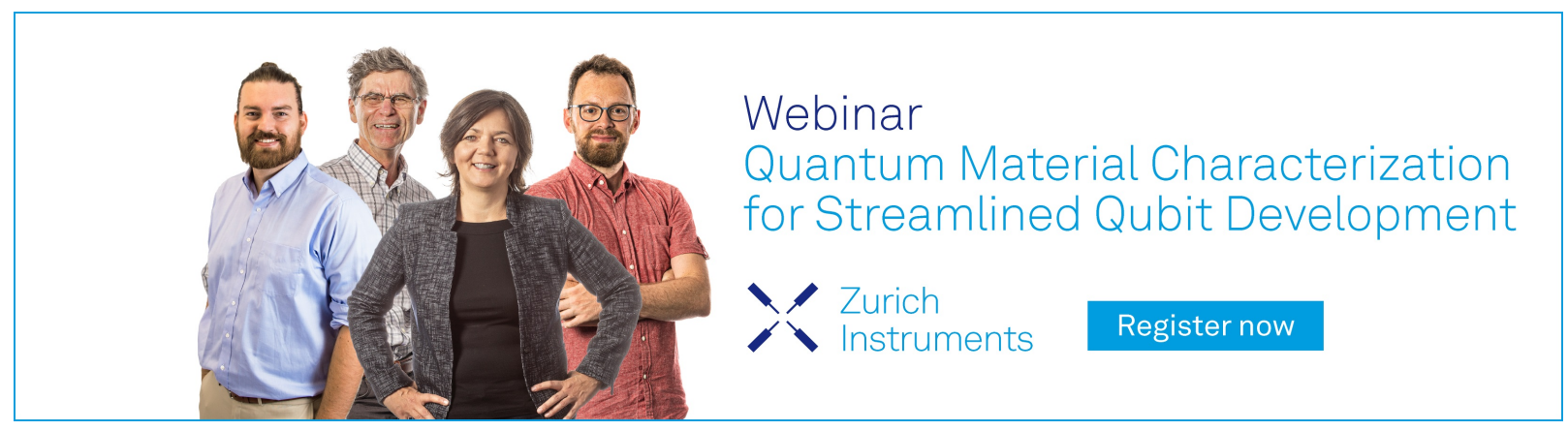




\title{
Domain wall stability in ferroelectrics with space charges
}

\author{
Yinan Zuo, ${ }^{\text {a) }}$ Yuri A. Genenko, Andreas Klein, Peter Stein, and Baixiang Xu \\ Institute of Materials Science, Technische Universität Darmstadt, D-64287 Darmstadt, Germany
}

(Received 22 November 2013; accepted 8 February 2014; published online 27 February 2014)

\begin{abstract}
Significant effect of semiconductor properties on domain configurations in ferroelectrics is demonstrated, especially in the case of doped materials. Phase field simulations are performed for ferroelectrics with space charges due to donors and electronic charge carriers. The free charges introduced thereby can act as a source for charge compensation at domain walls with uncompensated polarization bound charges. Results indicate that the equilibrium position of a domain wall with respect to its rotation in a head-to-head or a tail-to-tail domain configuration depends on the charge defect concentration and the Fermi level position. (C) 2014 AIP Publishing LLC.

[http://dx.doi.org/10.1063/1.4866359]
\end{abstract}

\section{INTRODUCTION}

Ferroelectric perovskites are widely used materials in actuators, sensors, and memory devices. They are wide gap semiconductors ${ }^{1}$ and are often treated as ideal dielectrics. However, oxygen vacancy is one kind of defect which is inevitable in ferroelectrics. Those defects can act as donors and give rise to ionic as well as electronic space charges. In this sense, the material behaves like a semiconductor.

Domain structures play a significant role in applications of ferroelectrics. Stability of domain structures in ferroelectrics is an important issue. A good estimation has been done for space charge layers in semiconducting ferroelectrics. ${ }^{1}$ Transmission optical microscopy shows that the domain walls in barium titanate are pinned after long time aging and thus, the domain structure is stabilized by presumably space charge. ${ }^{2}$ As it is known, two types of domain walls, i.e., $180^{\circ}$ and $90^{\circ}$ domain walls, are found as common equilibrium domain configurations of ferroelectrics free of space charges. Both of them are free of non-compensated polarization charges. However, in the case of ferroelectrics with free space charges, the otherwise unstable non-compensated domain walls can be stabilized. Several possibilities of domain structure stabilization have been discussed for Mn-doped barium titanate. ${ }^{3}$ First-principle calculations have also confirmed that by inserting charged atomic layers, head-to-head and tail-to-tail domain structures are stabilized. ${ }^{4}$ Gureev et al. showed that charged domain walls are energetically more favorable in comparison with the monodomain state of the ferroelectric and can make head-to-head and tail-to-tail domain structures stable. ${ }^{5}$ Mokrý et al. ${ }^{6}$ obtained an analytic solution of the pressure acting on a head-to-head domain wall and studied the influence of charge compensation and the external electric field. In a subsequent work Gureev et $a .^{7}$ also analytically studied the interaction of electric field with charged domain walls, which points out that the free charge carried by the wall depends on the size of the adjacent domains. A study of conductivity distribution across a charged domain wall has been performed for both

a)zuo@mfm.tu-darmstadt.de head-to-head and tail-to-tail domain configurations, and accumulation of electrons around head-to-head domain walls was predicted. ${ }^{8}$

On the aspect of formulation of domain wall motion, the idea of a generalized force acting on a defect traces back to Peach and Koehler ${ }^{9}$ for the study on dislocations in a crystal and Eshelby ${ }^{10,11}$ for the study on the inclusion problem in elasticity. Configurational forces present a basic concept of continuum physics and require their own balance. ${ }^{12}$ They serve as a powerful tool in identifying the driving force on an interface between phases. ${ }^{13}$ Motion of a discontinuity or a singularity in continua is characterized by the concept of configurational force and level set method. ${ }^{14}$ Budiansky and Rice have defined the path-independent integrals for rotation and expansion of inhomogeneities for infinitesimal elasticity. ${ }^{15}$ Eischen and Herrmann have made an attempt to connect the energy release rate and the path-independent integrals. ${ }^{16}$ By postulating balance laws, energy release rate and configurational moment were derived for a crack tip evolution by Agiasofitou and Kalpakides. ${ }^{17} \mathrm{Xu}$ et al. ${ }^{18}$ generalized the configurational force theory for ferroelectrics with domain structures and investigated the energy release rate at the crack tip in such materials.

In the current study, a phase field model is presented first in Sec. II for the study of the domain evolution in a barium titanate single crystal with space charges, formulated according to semiconducting features. In the model, we treat the spontaneous polarization as an order parameter, whose evolution subordinates to the Landau-Ginzburg equation in a thermodynamically consistent way. Phase field methods are used widely for the study of domain structures in ferroelectrics. ${ }^{18-28}$ Phase field modeling has also been applied to ferroelectrics with semiconducting features, for instance, the depletion layers near the electrodes were predicted and the interaction between the donors and the different domain structures were investigated. ${ }^{29,30}$ In a recent study, it was found that, with increased concentration of charged domain walls, the piezoelectric properties are enhanced. ${ }^{31}$ It has also been experimentally demonstrated that the charged head-to-head domain wall shows stable metallic-type conductivity. ${ }^{32}$ 
Two illustrative examples, depletion layers and head-tohead domain walls, are first studied in Sec. III to show the accumulation of free electronic charges near the electrodes and at non-compensated domain walls. For a quantitative study of stabilization of the domain walls with uncompensated polarization charges by free space charges, the concept of configurational force is applied. To start with, the driving force and moment along a domain wall between two headto-head hard domains is numerically calculated in Sec. IV. The effect of an external electric field, the domain wall orientation and the charge compensation on the tendency of motion of the domain wall is demonstrated. The results of the pressure on the domain wall agree well with those by Mokrý et al. ${ }^{6}$ However, the results reveal additionally a tendency of domain wall rotation besides the domain wall motion in the normal direction. The driving moment resulted from inhomogeneity of the electric field can affect the equilibrium position of the domain wall in a head-to-head or tail-to-tail domain configuration. This is then investigated by the phase field simulations in Sec. V. It is also shown that the equilibrium angle of the domain wall depends on the donor concentration.

\section{A PHASE FIELD MODEL FOR TETRAGONAL FERROELECTRICS}

Ferroelectrics are materials in which mechanical and electric phenomena take place in a coupled way. Thus, an appropriate model should involve both mechanics and electrostatics of solids, and the field variables take part in coupled constitutive laws. Even though the simulation examples considered in this paper are assumed to be stress free, a coupled model is presented here without loss of generality. The equilibria for stress and electric displacement have the form

$$
\begin{gathered}
\sigma_{i j, j}=0, \quad \text { in } \Omega, \\
D_{i, i}=\rho(\phi), \quad \text { in } \Omega,
\end{gathered}
$$

where $\Omega$ denotes the set of material points of the ferroelectrics in the space, $\sigma_{i j}$ is the Cauchy stress, $D_{i}$ is the electric displacement in the ferroelectrics, and $\rho(\phi)$ is the space charge density, the formulation of which will be explained later in this section. $(\cdot)_{, i}$ indicates $\partial(\cdot) / \partial x_{i}$ and Einstein summation convention is adopted. The volume force is neglected here. For both of the equilibrium equations, mechanical and electrostatic, either the Neumann or the Dirichlet boundary condition can be imposed. The infinitesimal strain $S_{i j}$ is taken as the symmetric part of the gradient of displacement field $u_{i}$, and the electric field $E_{i}$ as the negative gradient of the electric potential $\phi$. That is,

$$
\begin{gathered}
S_{i j}=\frac{1}{2}\left(u_{i, j}+u_{j, i}\right), \\
E_{i}=-\phi_{, i} .
\end{gathered}
$$

\section{A. The constitutive laws and the evolution equation}

The total Gibbs energy $H$ is comprised of three parts

$$
H=H^{\text {ent }}\left(S_{i j}, E_{i}, P_{i}\right)+H^{\text {sep }}\left(P_{i}\right)+H^{\text {grad }}\left(P_{i, j}\right),
$$

in which the term $H^{\text {ent }}$ takes the form

$$
\begin{aligned}
H^{e n t}= & \frac{1}{2}\left(S_{i j}-S_{i j}^{0}\right) C_{i j k l}\left(S_{k l}-S_{k l}^{0}\right)-\left(S_{i j}-S_{i j}^{0}\right) b_{k i j} E_{k} \\
& -\frac{1}{2} E_{i} \varepsilon_{i j} E_{j}-P_{i} E_{i},
\end{aligned}
$$

where $C_{i j k l}$ is the elastic stiffness tensor, $S_{i j}^{0}$ is the eigenstrain introduced by the spontaneous polarization in tetragonal phase, $b_{i j k}$ denotes the piezoelectric tensor, $\varepsilon_{i j}$ is the permittivity tensor, and $P_{i}$ is the spontaneous polarization. The electrostrictive coupling is considered through the dependence of spontaneous strain on the polarization. In fact, expansion of the first term in Eq. (6) and insertion of a quadratic dependence of the spontaneous strain on the polarization lead to an electrostriction term $q_{i j m n} S_{i j} P_{m} P_{n}$, known in the phase field models, e.g., by $\mathrm{Chen}^{33}$ and Sluka et al. ${ }^{31}$ It should be noted that in our model, we adopted a more complex nonlinear dependence of $S_{i j}^{0}$ on the polarization introduced by Kamlah, ${ }^{34}$ but the argument for the electrostrictive coupling remains the same. Note that the tensor $b_{i j k}$ depends also nonlinearly on the polarization. For more details on their dependence, the readers are referred to our previous work. ${ }^{23,35}$ Moreover, for ferroelectrics with a centrosymmetric parent phase which is the case in our problem, a bi-linear term of the spontaneous polarization and the strain should be absent. ${ }^{33,36}$

$H^{\text {sep }}$ is an even polynomial function of the spontaneous polarization components as the Landau energy. In the 2D case,

$$
\begin{aligned}
H^{s e p}= & \frac{\kappa_{s} G}{\epsilon}\left[a_{1}+a_{2}\left(P_{1}^{2}+P_{2}^{2}\right)+a_{3}\left(P_{1}^{4}+P_{2}^{4}\right)\right. \\
& \left.+a_{4} P_{1}^{2} P_{2}^{2}+a_{5}\left(P_{1}^{6}+P_{2}^{6}\right)\right] .
\end{aligned}
$$

Here, $a_{i}(i=1,2, \ldots, 5)$ are parameters which can be found in the previous work. ${ }^{37}$ The polynomial function imposes a four-well energy landscape, with four local minima corresponding to the four domain variants. The reduced sixth order polynomial is used here, so that the energy landscape can be fully determined by the extreme points indicating domain states and the information of the saddle points between $90^{\circ}$ domain switching. Note that the coefficient $a_{1}$ is chosen in such a way that the energy of the uniformly poled state with a spontaneous polarization for the considered temperature is subtracted. In this way, Eq. (7) is only related to the nonuniform part of the energy associated with the polarization. In other words, it works as a penalty function, which ensures that the spontaneous polarization in the domain interior takes one of the four domain variants. The parameters $G$ and $\epsilon$ are the specific energy and the width of the domain wall, respectively. The coefficient $\kappa_{s}$, together with the coefficient $\kappa_{i}$ introduced later, is used to calibrate the parameters $G$ and $\epsilon$. With the calibration coefficients $\kappa_{s}$ and $\kappa_{i}$, the material parameters $G$ and $\epsilon$ coincide with the domain wall energy and the domain wall width of a $180^{\circ}$ domain wall. More details on this calibration approach can be found in 
works. $^{35,38}$ The term $H^{\text {grad }}$ is expressed by the norm of the gradient of the polarization vector. For the 2D case,

$$
H^{\text {grad }}=\kappa_{i} \frac{G \epsilon}{P_{0}^{2}}\left(P_{1,1}^{2}+P_{1,2}^{2}+P_{2,1}^{2}+P_{2,2}^{2}\right) .
$$

The coupled constitutive relationships are then derived from

$$
\begin{gathered}
\sigma_{i j}=\frac{\partial H}{\partial S_{i j}}=C_{i j k l}\left(S_{k l}-S_{k l}^{0}\right)-b_{k i j} E_{k}, \\
D_{i}=-\frac{\partial H}{\partial E_{i}}=b_{i j k}\left(S_{j k}-S_{j k}^{0}\right)+\varepsilon_{i j} E_{j}+P_{i} .
\end{gathered}
$$

It is seen that the total polarization consists of three parts: the reversible contribution by the elastic strain, the reversible contribution by the electric field, and the spontaneous part. The spontaneous polarization is irreversible and results from minimization of the total free energy. This interpretation of the order parameter coincides with that by Landau and Lifshitz ${ }^{36}$ and that by Sluka et al. ${ }^{31}$ The reversible contribution by the electric field to the electric displacement was also included in the model by Sluka et al., ${ }^{31}$ whereas the reversible contribution by the elastic strain is additionally considered in the present model.

As a result of the thermodynamic law, a LandauGinzburg type equation is obtained for the evolution of the spontaneous polarization

$$
\frac{\partial P_{i}}{\partial t}=-M \frac{\delta H}{\delta P_{i}}=-M\left(\frac{\partial H^{e n t}}{\partial P_{i}}+\frac{\partial H^{s e p}}{\partial P_{i}}-2 \kappa_{i} \frac{G \epsilon}{P_{0}^{2}} P_{i, k k}\right),
$$

where $M$ is the domain wall mobility parameter and $\delta(\cdot) / \delta(\cdot)$ denotes the functional derivative. The readers are referred to the previous work $^{23,35}$ for more details on the model and its numerical implementation in the finite element method (FEM). The values for the involved parameters can be found in Table I.

In summary, there are three major features in the current phase field model. First, instead of the total polarization, the spontaneous polarization is treated as the order parameter. Second, the polynomial function in the energy plays a role of penalty. Finally, it allows us to directly include the two physical parameters, i.e., the domain wall energy and the domain wall thickness, into the model.

\section{B. Space charge in ferroelectrics with semiconducting features}

In dielectrics, both dopant atoms and intrinsic point defects can be donors and acceptors which make the material an n-type or p-type semiconductor. In particular, oxygen vacancies are common point defects, whose appearance is inevitable in the device production and working cycles. Depending on the electric potential, a certain portion of donors or acceptors is ionized and the electrons or holes are available. According to the general semiconducting theory, the local space charge consists of four parts ${ }^{39}$

$$
\begin{aligned}
\rho(\phi)= & -q N_{C} F_{1 / 2}\left(\frac{E_{F}-E_{C}+q \phi}{k T}\right) \\
& +q N_{V} F_{1 / 2}\left(\frac{E_{V}-E_{F}-q \phi}{k T}\right) \\
& +q z_{d} N_{d} t_{d}(\phi)-q z_{a} N_{a} t_{a}(\phi),
\end{aligned}
$$

\begin{tabular}{|c|c|c|c|c|c|}
\hline Parametert & Value & Parameter & Value & Parameter & Value \\
\hline$C_{11}$ & $14 \times 10^{10} \mathrm{Nm}^{-2}$ & $A_{12}$ & 0.0 & $P_{0}$ & $0.4 \mathrm{Cm}^{-2}$ \\
\hline$C_{12}$ & $1.4 \times 10^{10} \mathrm{Nm}^{-2}$ & $A_{21}$ & 0.0 & $S_{0}$ & 0.003 \\
\hline$C_{13}$ & 0.0 & $A_{22}$ & $6.0 \times 10^{-9} \mathrm{CV}^{-1} \mathrm{~m}^{-1}$ & $E_{F}$ & $-5.300 \mathrm{eV}$ \\
\hline$C_{21}$ & $1.4 \times 10^{10} \mathrm{Nm}^{-2}$ & $M$ & $1.0 \times 10^{3} \mathrm{AV}^{-1} \mathrm{~m}^{-1}$ & $E_{C}$ & $-3.600 \mathrm{eV}$ \\
\hline$C_{22}$ & $34 \times 10^{10} \mathrm{Nm}^{-2}$ & $G$ & $0.067 \mathrm{Jm}^{-2}$ & $E_{V}$ & $-6.600 \mathrm{eV}$ \\
\hline$C_{23}$ & 0.0 & $\epsilon$ & $1.6 \times 10^{-7} \mathrm{~m}$ & $E_{d}$ & $-4.000 \mathrm{eV}$ \\
\hline$C_{31}$ & 0.0 & $\kappa_{s}$ & 0.70 & $E_{a}$ & $-6.200 \mathrm{eV}$ \\
\hline$C_{32}$ & 0.0 & $\kappa_{i}$ & 0.17 & $z_{d}$ & 2 \\
\hline$C_{33}$ & $16 \times 10^{10} \mathrm{Nm}^{-2}$ & $a_{1}$ & 1.0 & $z_{a}$ & 2 \\
\hline$b_{11}$ & 0.0 & $a_{2}$ & $5.824 \times 10^{-2} \mathrm{~m}^{4} \mathrm{C}^{-2}$ & $g_{d}$ & 2 \\
\hline$b_{12}$ & 0.0 & $a_{3}$ & $-117.9 \mathrm{~m}^{8} \mathrm{C}^{-4}$ & $g_{a}$ & $1 / 2$ \\
\hline$b_{13}$ & $13.0 \mathrm{Cm}^{-2}$ & $a_{4}$ & $214.4 \mathrm{~m}^{8} \mathrm{C}^{-4}$ & $N_{C}$ & $10^{24} \mathrm{~m}^{-3}$ \\
\hline$b_{21}$ & $-5.2 \mathrm{Cm}^{-2}$ & $a_{5}$ & $490.6 \mathrm{~m}^{12} \mathrm{C}^{-6}$ & $N_{V}$ & $10^{24} \mathrm{~m}^{-3}$ \\
\hline$b_{22}$ & $-1.5 \mathrm{Cm}^{-2}$ & $q$ & $1.6022 \times 10^{-19} \mathrm{C}$ & $N_{d}^{42}$ & \\
\hline$b_{23}$ & 0.0 & $k_{B}$ & $1.3807 \times 10^{-23} \mathrm{~J}$ & $N_{a}$ & 0 \\
\hline$A_{11}$ & $6.0 \times 10^{-9} \mathrm{CV}^{-1} \mathrm{~m}^{-1}$ & $T$ & $300 \mathrm{~K}$ & & \\
\hline
\end{tabular}

in which

$$
\begin{aligned}
& t_{d}(\phi)=1-\frac{1}{1+\frac{1}{g_{d}} \exp \left(\frac{E_{d}-E_{F}-q \phi}{k T}\right)}, \\
& t_{a}(\phi)=1-\frac{1}{1+g_{a} \exp \left(\frac{E_{a}-E_{F}-q \phi}{k T}\right)} .
\end{aligned}
$$

TABLE I Parameters used in the simulations. 


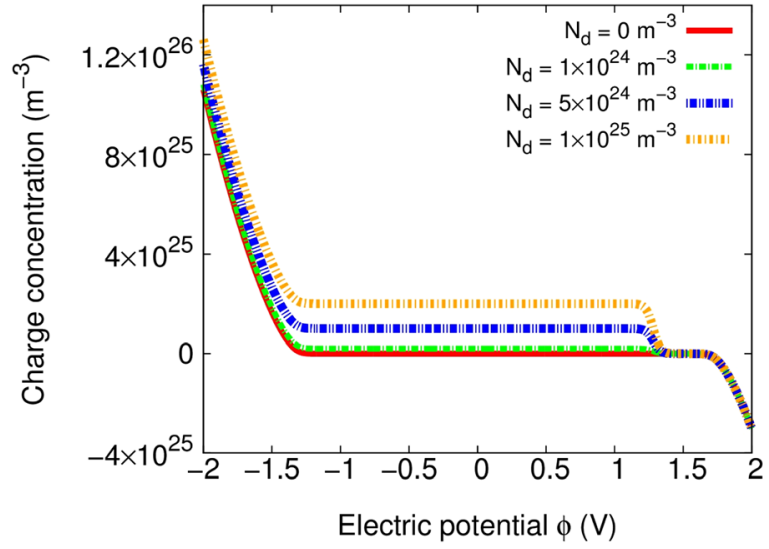

FIG. 1. Variation of the space charge with respect to the electric potential in ferroelectrics with semiconducting features according to Eq. (12) with $N_{a}=0$.

In the equations, $q$ is the electron charge, $N_{C}$ and $N_{V}$ are effective densities of states in the conduction band and the valence band, and $E_{F}$ is the Fermi level. According to the work by Xiao et al., ${ }^{29}$ the Fermi level in the material is taken as the Fermi level of the electrode, for instance, the platinum electrode. $E_{C}$ and $E_{V}$ are the conduction and the valence band edges, respectively. $N_{d}$ and $N_{a}$ are the concentrations of donors and acceptors, whose ionized portions are denoted by $t_{d}$ and $t_{a} . z_{d}$ and $z_{a}$ are the donor valency and the acceptor valency, respectively. $k$ is the Boltzmann constant, $T$ is the absolute temperature, and $F_{1 / 2}$ is the Fermi integral. The Fermi integral is numerically calculated according to the scheme by van Halen and Pulfrey. ${ }^{40}$ Thus, space charge density is coupled with acceptor and donor concentrations, electric potential and temperature at the same time. In this study, no acceptor is considered and it is assumed that the donors have a homogeneous spatial distribution and possess no mobility. The values of parameters used in the simulation are listed in Table I according to the Ref. 29. Note that $z_{d}$ is set to be 2 here, instead of 1 . It was demonstrated in first principle calculations that in barium titanate doubly charged oxygen vacancies are dominant in the $n$-type region. ${ }^{41}$

The space charge density is plotted as a function of electric potential in Fig. 1. For defect-free barium titanate, the material remains space charge neutral in the range of $-1.30 \mathrm{~V}<\phi<1.70 \mathrm{~V}$, which corresponds to $\left(E_{V}-E_{F}\right) /$ $q<\phi<\left(E_{C}-E_{F}\right) / q$. For $\phi>1.70 \mathrm{~V}$, a large amount of electrons move to the conducting band, whereas for $\phi<-1.30 \mathrm{~V}$, holes move into the valence band. After introducing oxygen vacancies as donors, a sufficiently low electric potential favors the ionization and thus positive charge carriers dominate. From Fig. 1, it is seen that the potential difference between the commence of electron jumping into the conduction band and the point when $1 / 3$ of donors are ionized is about $0.4 \mathrm{~V}$. This value corresponds to $\left(E_{C}-E_{D}\right) / q$. The space charge density due to the ionization of donors is proportional to the donor concentration.

\section{FREE CHARGE ACCUMULATION}

\section{A. Depletion layer}

As an example to demonstrate the formation of space charges, a periodic domain structure in a barium titanate thin

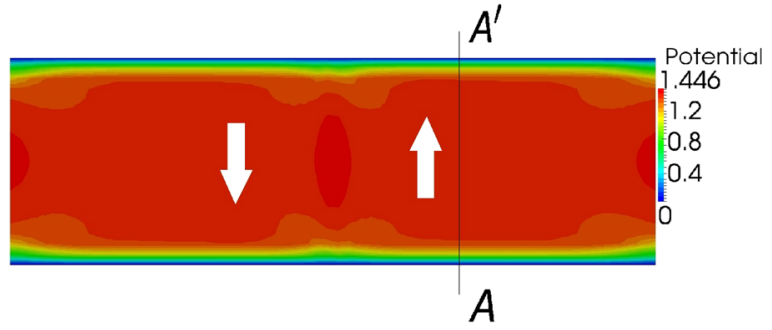

FIG. 2. Distribution of the electric potential in a barium titanate film with donor concentration of $N_{d}=5 \times 10^{24} \mathrm{~m}^{-3}$. The upper and lower surfaces are assumed to be potential-free, corresponding to a short circuit condition.

film sandwiched between two platinum electrodes is studied. Results show that the charge carriers diffuse away in a certain thickness near the electrodes, giving rise to a depletion layer (Fig. 2). A built-in potential arises in the interior of the material slab, and the charge density increases in the depletion layer from interior to the edge (Fig. 3). This result indicates that the sample is positively charged. This should be due to the asymmetry of the defect system considered here. Likewise, an acceptor-dominant sample was shown to be negatively charged in the work by Genenko et al. ${ }^{43}$ Donors are almost fully ionized in the depletion layers, whereas the ionized proportion of donors is only about $10 \%$ in the interior. Those results are consistent with the features demonstrated in Fig. 1.

The width of the depletion layer can vary with the concentration of donors (Fig. 4). The larger the concentration is, the thinner the depletion layer becomes. This is related to the fact that, for larger concentration of donors, the distance to be covered in order to build up certain value of electric potential is smaller. The built-in potential can modify the polarization distribution to a certain extent. The polarization component increases on the side favored by the electric field due to the built-in potential, whereas the component decreases on the other side (Fig. 5). These results are in agreement with those by Xiao et $_{\text {al. }}{ }^{29}$

\section{B. Stabilization of head-to-head domain structure}

The head-to-head domain configuration is generally not a stable one in a non-semiconducting case, since the

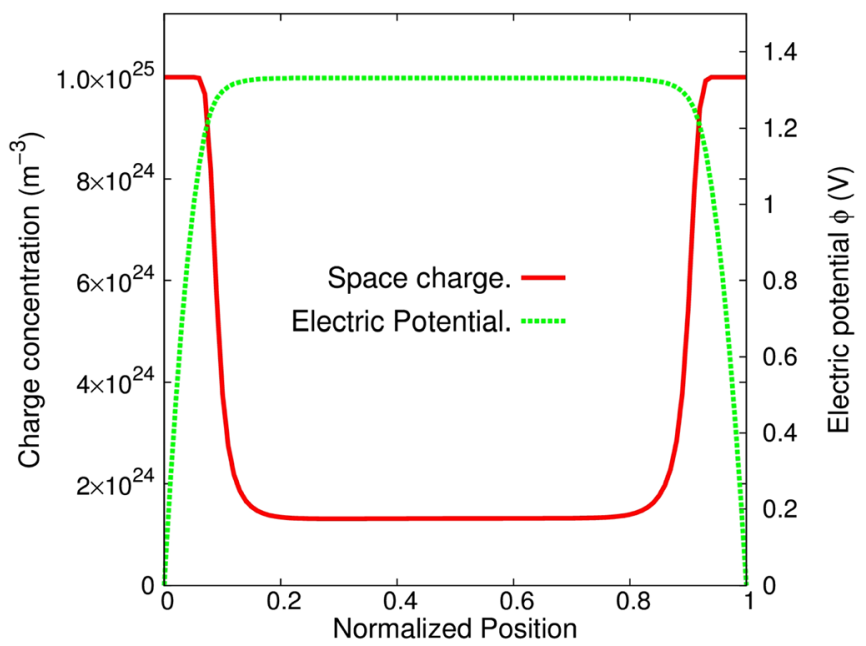

FIG. 3. Distribution of the electric potential and of the space charge along the cross section $\mathrm{AA}^{\prime}$ depicted in Fig. 2. 


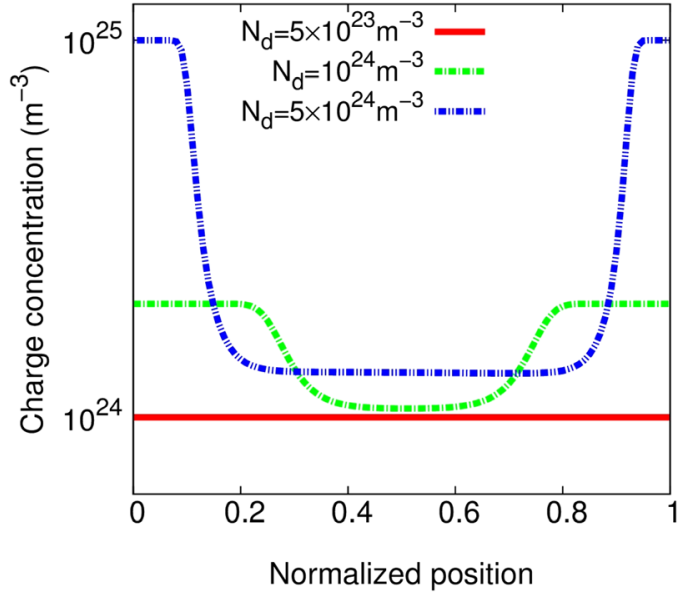

FIG. 4. The formed depletion layers for different concentrations of donors.

non-compensated charges due to the polarization lead to high energy density and result in a driving force on the domain wall. However, when the free space charge is available, the domain configuration can be stabilized.

In this subsection, the phase field model is applied to study the evolution of a $180^{\circ}$ head-to-head domain wall for the cases with and without semiconducting features, respectively. Fig. 6(a) illustrates the initial configuration for the simulations. The left and right edges are short circuited, i.e., the electric boundary condition for the two edges are set to be potential-free, whereas the system is periodically extended in the vertical direction. All the boundaries are supposed to be traction-free. Fig. 6(b) shows the obtained equilibrium domain configuration for the case that the space charge due to the semiconducting features is excluded. This lamellar domain structure is virtually charge-free. Unlike the phase field modeling by Wang and Kamlah, ${ }^{44}$ where the domain configuration in a ferroelectric nanotube was manipulated by prescribed surface charge density, the lamellar domain structure arises here due to a periodical boundary setup.

On the other hand, if the space charge of semiconducting nature is considered, the obtained equilibrium domain configuration remains almost the same as the initial one (Fig. 6(c)). The domain wall becomes simply thicker, as suggested by experimental observations. ${ }^{45}$ Results indicate that the uncompensated polarization charges along the initial domain wall are now compensated by the free space charges from

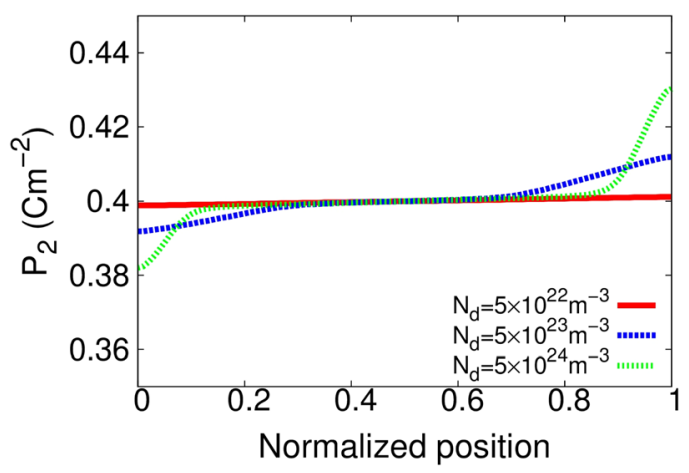

FIG. 5. Variation of $P_{2}$ due to the built-in potential.

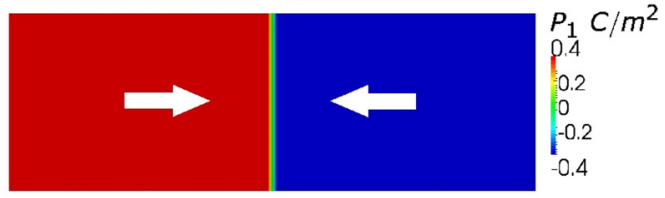

(a)

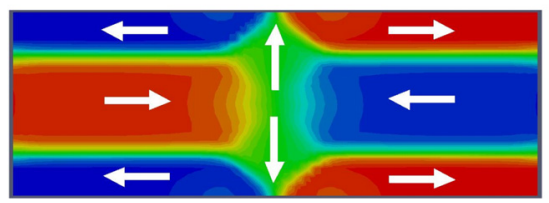

(b)

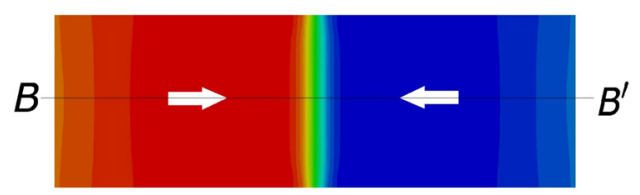

(c)

FIG. 6. (a) The initial head-to-head domain configuration. (b) The equilibrium domain configuration without semiconducting features. (c) The equilibrium domain configuration with a donor concentration of $N_{d}=5 \times 10^{23} \mathrm{~m}^{-3}$.

the semiconducting material (Fig. 7). In Fig. 7, the energy band of the electrons is also demonstrated. In this way the electrostatic energy due to the uncompensated polarization charges is counteracted by the contribution of the semiconductor space charges. This may also be justified by the distribution of the resultant electric potential and of the resultant polarization presented in Fig. 8.

\section{DRIVING FORCE AND DRIVING MOMENT ALONG A DOMAIN WALL BETWEEN TWO HEAD-TO-HEAD HARD DOMAINS}

This section is devoted to a quantitative study of the stability of uncompensated domain walls and the influence of the space charges. To start with, we consider a domain wall between two hard domains with head-to-head polarization configuration, illustrated in Fig. 9. The driving force acting

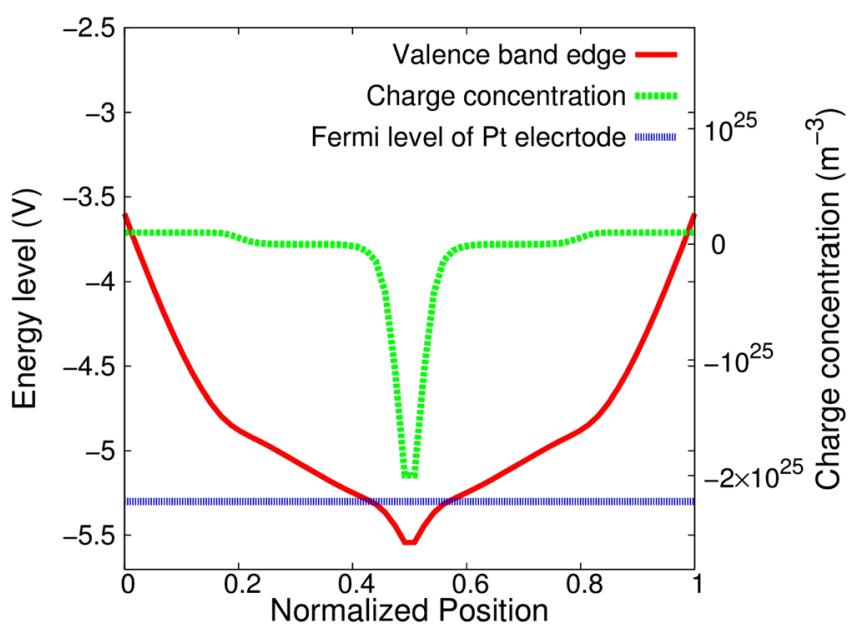

FIG. 7. Distribution of the conduction band edge and of the space charge along the cross section $\mathrm{BB}^{\prime}$ highlighted in Fig. 6 . The donor concentration in this example is $N_{d}=5 \times 10^{23} \mathrm{~m}^{-3}$. 


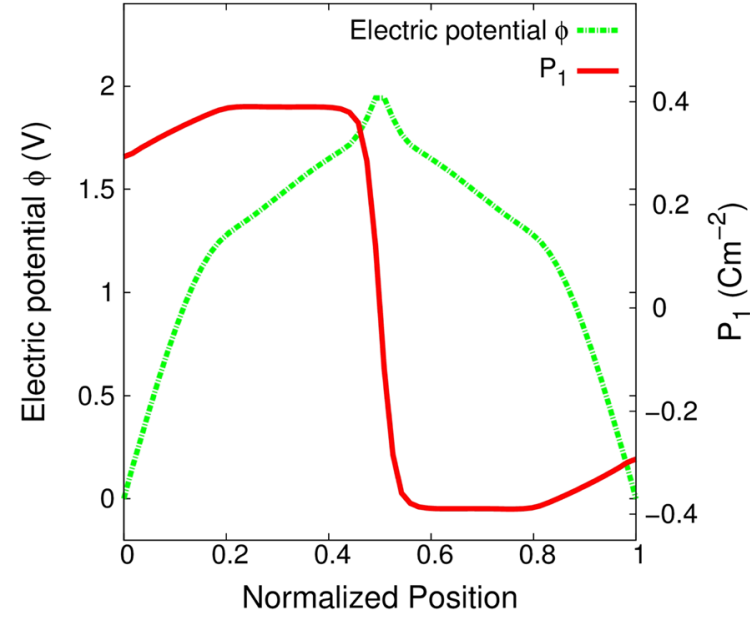

FIG. 8. Variation of the electric potential and of the horizontal polarization component $P_{1}$ along the cross section $\mathrm{BB}^{\prime}$ highlighted in Fig. 6 . The donor concentration in this example is $N_{d}=5 \times 10^{23} \mathrm{~m}^{-3}$.

on the domain wall will be computed through numerically calculated nodal configurational forces.

According to Maugin, ${ }^{46}$ the Eshelby tensor for piezoelectricity has the form

$$
\Sigma_{i j}=\Psi \delta_{i j}-u_{k, i} \sigma_{k j}+D_{i} E_{j},
$$

where $\Psi$ denotes the free energy of the system expressed by Eq. (6). The corresponding configurational force vector is

$$
g_{i}=-u_{j, i} f_{j}+\rho \phi_{, i}
$$

where $f_{i}$ is the volume force which is neglected in this study. The condition

$$
\Sigma_{i j, j}+g_{i}=0,
$$

must hold. That is, $\Sigma_{i j}$ and $g_{i}$ correlate with each other in a similar way as the Cauchy stress tensor and the volume force do. For a dielectric solid with an interface between two phases, $\Sigma_{i j}$ experiences a jump across the interface. The configurational force vector $g_{i}$ is then concentrated at the interface, which can be interpreted as the driving force on the interface. For a selected control volume $V_{c}$, the overall

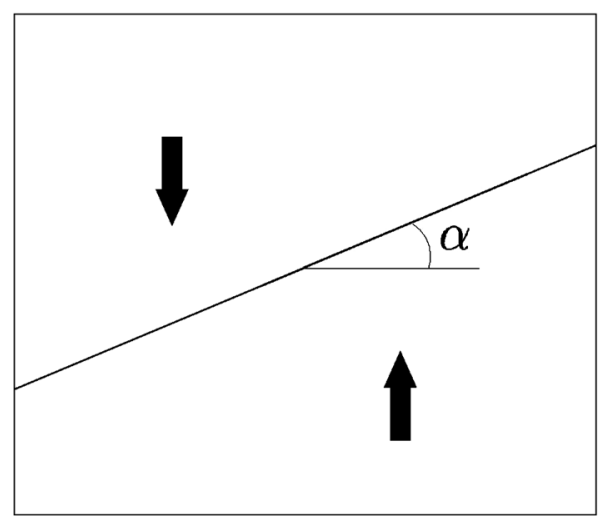

FIG. 9. An illustrative sketch of a head-to-head domain configuration with the domain wall angle $\alpha$. driving force and driving moment on the interface which is circumvented in the control volume $V_{c}$, are given by ${ }^{12,15}$

$$
\begin{gathered}
J_{i}=-\int_{V_{c}} g_{i} d V, \\
L_{i}=-\int_{V_{c}} \epsilon_{j k i} x_{j} g_{k} d V,
\end{gathered}
$$

where $x_{i}$ is the location vector of the material point and $\epsilon_{i j k}$ denotes the Levi-Civita symbol. The volume integral may be rewritten into a path integral around the control volume according to the Gauss' theorem. For the considered headto-head domain configuration in Fig. 9, the integral path can be chosen as any path which circumvents the whole domain wall. Since there is no inhomogeneity inside both of the domains, no configurational force will appear in the interior of the domains. The configuration force appears merely along the domain wall. Therefore, one can simply take the line along the domain wall as the integral path.

In the FEM simulation, $\Sigma_{i j}$ is calculated from the obtained field quantities, according to Eq. (15). The configurational force $g_{i}$ at one element node is then calculated by summing up all the contributions from all adjacent elements around the node. Without going into too many details, the nodal driving force $G_{i}^{I}$ at the node I is given by ${ }^{47,48}$

$$
G_{i}^{I}=\bigcup_{e=1}^{n_{e}} \int_{\Omega_{e}} \Sigma_{i j} N_{. j}^{I} d \Omega
$$

where the assembly operation is performed over all elements adjacent to node I, $\Omega_{e}$ is the element area of the $e$-th element, $n_{e l}$ is the number of the elements around the node $\mathrm{I}$, and $N^{I}$ is the shape function associated with the node I in the corresponding element. The derivative of the shape functions $N^{I}$ can be computed in the same manner as for standard finite element techniques.

The above mentioned approach for computing the nodal configurational force is now applied to the domain configuration shown in Fig. 9. In the considered head-to-head domain configuration, the domain wall orientation is characterized by the angle $\alpha$ with respect to the horizontal. In this evaluation, the mechanical aspects are neglected, since we focus on a mechanical stress-free situation. Because the component of the configurational force tangential to the domain wall induces no motion, the normal component (the driving force) will be particularly discussed. Depending on the external loading and the charge compensation, the following four cases are studied separately.

Case 1: Without external electric field and charge compensation.

In this case, the top and the bottom surfaces are set to be potential-free. For $\alpha=0^{\circ}$, the driving force is zero everywhere along the domain wall. The system resides in an equilibrium but energetically unfavorable state. Therefore, it is not stable and will develop into a more stable state if the polarization is free to evolve, as the example in Sec. III demonstrates. For $0^{\circ}<\alpha<90^{\circ}$, there is a resultant configurational moment on the interface (Fig. 10), due to the 


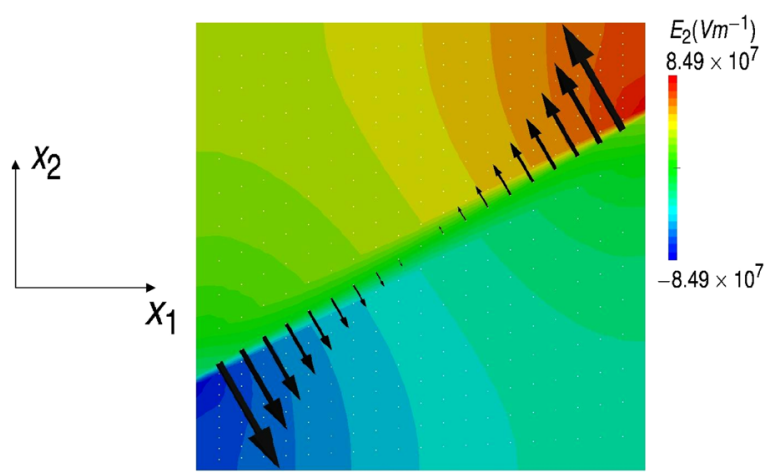

FIG. 10. Distribution of the vertical component of the electric field and of the configurational force vector along the domain wall with the angle $\alpha=30^{\circ}$. This plot is for Case 1 , namely, without applied electric field and charge compensation.

inhomogeneity of the electric field resulting from the uncompensated polarization charges. This moment implies that the domain wall intends to rotate to the vertical position to minimize the total energy of the system, as it will be demonstrated by the phase field simulations in Sec. V. The dependence of the resultant driving moment on the angle $\alpha$ is plotted in Fig. 11. This moment reaches its maximum at about $\alpha=45^{\circ}$. For $\alpha=90^{\circ}$, there is no uncompensated polarization charge and thus no electric field. This explains why the driving force for the vertical domain wall is zero along the domain wall.

Case 2: Without external electric field but with full charge compensation.

It is assumed in this case that the charges on the sharp interface due to the polarization are fully compensated, e.g., by free charge carriers available in the system. Since the domain wall is fully compensated by free charge carriers, there is no induced internal electric field for any angle $\alpha$. Thus, in absence of the external field, there is no driving force in the considered area.

Case 3: With external electric field but without charge compensation.

When the sample is subjected to an electric field of $10^{7} \mathrm{~V} / \mathrm{m}$ in the vertical direction, a distribution of driving force is induced along the domain wall, see Fig. 12. The

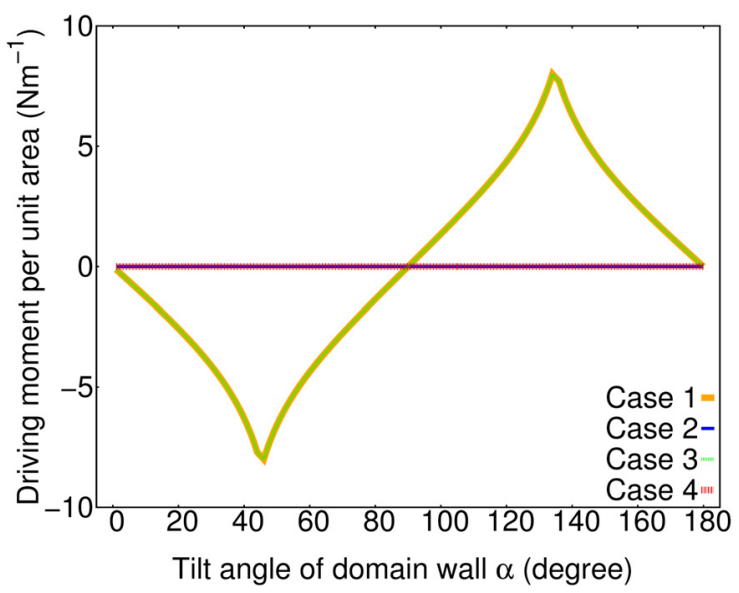

FIG. 11. Driving moment per unit area on the domain wall vs the domain wall tilt angle for the cases $1-4$.

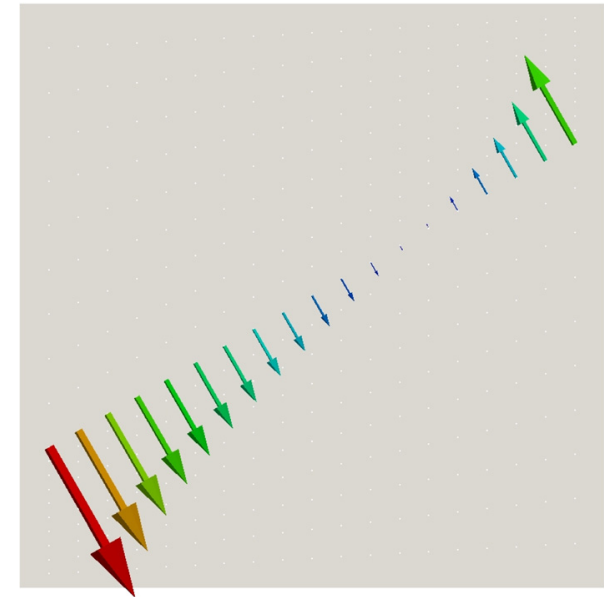

FIG. 12. Distribution of the configurational force vector along the domain wall with $\alpha=30^{\circ}$. This is for Case 3, namely, with an applied external electric field and no charge compensation.

driving force favors the growth of the domain whose polarization takes the same direction as the applied electric field. The average of the calculated driving force on the domain wall agrees with the analytical solution for the pressure on the domain wall obtained by Mokrý et al. ${ }^{6}$ The average driving force is also independent of the angle $\alpha$ (Fig. 13). This phenomenon was also observed in the work of Mokrý et al. ${ }^{6}$ On the other hand, due to the fact that the uncompensated polarization charges induce also inhomogeneous internal electric field, the driving force along the domain wall should not be homogeneous. Therefore, there is a variation of the driving force along the domain wall (Fig. 12). Subtracting the driving force by its average value, one can obtain the variation in the same fashion as that in Fig. 10 for Case 1. Therefore, there should exist a driving moment, which takes its maximum at the angle $45^{\circ}$. As it can be seen in Fig. 11, the dependence of the resultant moment on the angle $\alpha$ is exactly the same for both Case 1 and Case 3.

Case 4: With external electric field and full charge compensation.

Similar to Case 2, full charge compensation implies no internal electric field. The driving force is merely due to the

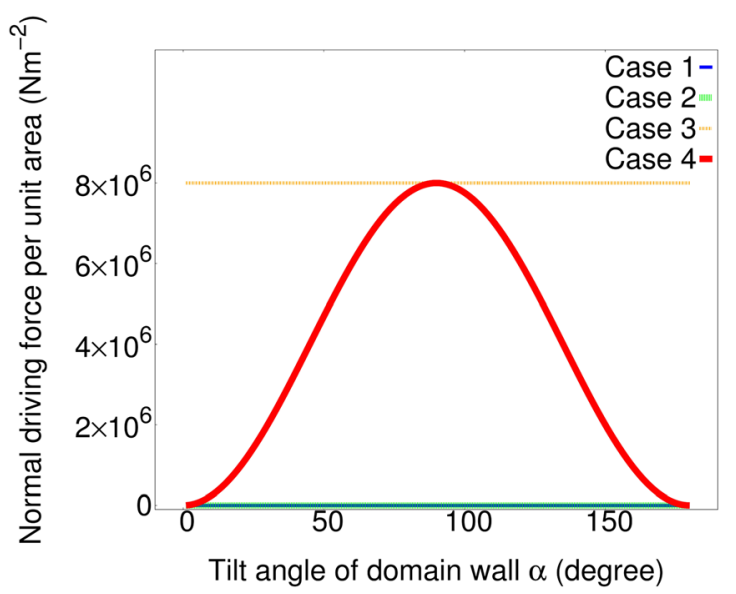

FIG. 13. Normal driving force per unit area vs. the domain wall tilt angle for the cases $1-4$. 
TABLE II. Summary of the results on the driving moment and the driving force for the cases $1-4$.

\begin{tabular}{lccc}
\hline \hline & With electric field & Without electric field \\
\hline $\begin{array}{l}\text { Full charge compensation } \\
\text { No charge compensation }\end{array}$ & $\begin{array}{c}\text { Orientation dependent driving force, no driving moment } \\
\text { Constant driving force, orientation dependent driving moment }\end{array}$ & No driving force, no driving moment \\
\hline
\end{tabular}

external electric field. In other words, the driving force remains uniform along the domain wall, and there is no driving moment. The driving force has a trigonometric dependence on the angle $\alpha$, as it is shown in Fig. 13. If $\alpha=0^{\circ}$, there is no driving force on the interface, indicating a stable domain structure. When $\alpha=90^{\circ}$, the driving force takes the same value as the average value of the driving force in Case 3. For other angle $\alpha$, besides the normal driving force there is a tangential component along the interface, which, however, does not play a role in the domain wall motion. An example of a partly compensated interface has also been studied, which appears as an intermediate situation between the case with full compensation and the one without compensation.

It is seen from Figs. 11 and 13 that the external electric field is responsible for the average normal driving force on the domain wall, whereas the inhomogeneity of the internal electric field induced by the uncompensated polarization charges causes the resultant driving moment on the domain wall. The situation of the driving force and moment for the four cases is summed up in Table II.

Note that the force acting on the above considered charged wall is by one order of the magnitude larger than the inhomogeneous clamping pressure exerted upon an uncharged domain wall due to the migration of oxygen vacancies. ${ }^{49,50}$ These two forces become, however, comparable for the applied electric fields of the order of the internal bias field $E_{i b} \simeq 10^{6} \mathrm{~V} / \mathrm{m}$.

Principal properties of driving forces and moments exerted upon hard domain walls were investigated in this section assuming their zero thickness that may lead to unphysical discontinuities at small scales. Phase field simulations presented in Sec. V, consider spatial variation of polarization that relaxes the above difficulty to some extent. Nevertheless, the used continuum mechanics approach remains limited by characteristic lengths considerably exceeding interatomic distances.

\section{ROTATION OF DOMAIN WALL TO THE EQUILIBRIUM AND ITS STABILIZATION BY SPACE CHARGES}

Phase field simulations based on the model presented in Sec. II are now performed to verify the domain wall rotation predicted in Sec. IV and its stabilization by space charges. An initial tail-to-tail domain configuration with $\alpha=60^{\circ}$ is used. Three different examples are considered: ferroelectrics free of semiconducting features, ferroelectrics with $N_{d}=10^{24} \mathrm{~m}^{-3}$, and $N_{d}=10^{25} \mathrm{~m}^{-3}$. All examples are with short circuit boundary condition, i.e., zero potential is prescribed on the top and the bottom surfaces, while the right and left edges are charge-free.

For dielectric ferroelectrics where no space charge is available, the domain wall is charged. The driving moment leads to rotation of the domain wall. As Fig. 14(a) shows, at equilibrium the domain wall reaches the vertical position in order to avoid a charged domain configuration.

Due to the tail-to-tail domain configuration, a low electric potential arises in the interior $(\phi<-1.3 \mathrm{~V})$, and thus, for a ferroelectric with $N_{d}=10^{24} \mathrm{~m}^{-3}$, a high positive charge density develops. As a result of evolution, the potential in the interior increases and an almost uniform charge density appears. The domain wall reaches a position between the initial one and the vertical one at equilibrium, see Fig. 14(b). In the case of $N_{d}=10^{25} \mathrm{~m}^{-3}$, an electric potential in the vicinity of the tilted domain wall lower than that in the domain interior is accompanied by a high charge density, so that almost no domain wall motion occurs (Fig. 14(c)). Thus, homogeneously distributed donors like oxygen vacancies can also hinder the domain wall rotation, providing a sort of imprint. 6,51

The rotation angle of the domain wall, from the initial configuration with $\alpha$ to its equilibrium state, is plotted with respect to the donor concentration in Fig. 15.

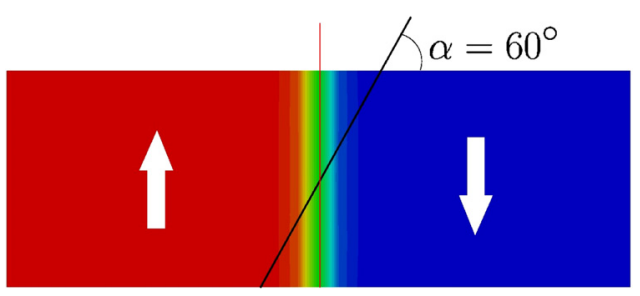

(a)

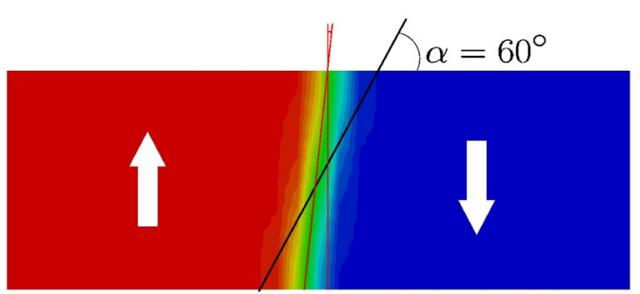

(b)

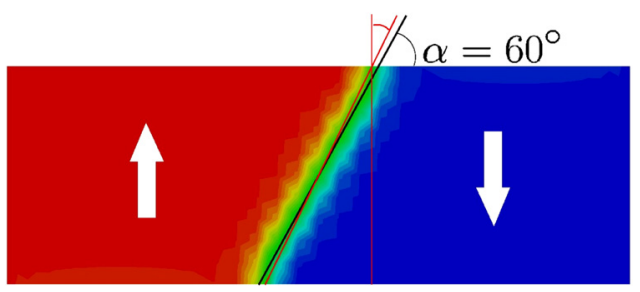

(c)

FIG. 14. Rotation of the domain wall as a result of the driving moment. The equilibrium domain configurations for (a) No semiconducting feature, (b) $N_{d}=10^{24} \mathrm{~m}^{-3}$, and (c) $N_{d}=10^{25} \mathrm{~m}^{-3}$, respectively. The black line denotes the initial position of the domain wall. 


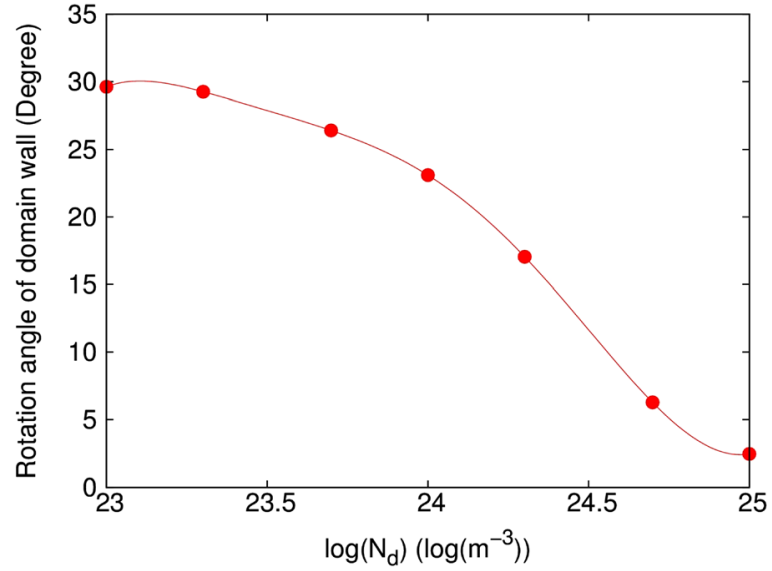

FIG. 15. The rotation angle of the domain wall after it reaches equilibrium.

The variations of the total free energy and the driving moment during the domain evolution for three different examples are plotted in Figure 16. For the nonsemiconducting case and the case of $N_{d}=10^{24} \mathrm{~m}^{-3}$, both the total free energy and the driving moment experience a sharp decrease at the beginning. As the evolution proceeds, the decrease of the free energy becomes slower and the configurational moment decreases. After reaching equilibrium, there is no configurational moment any more, and the free energy reaches its minimum. For the case of $N_{d}=10^{25} \mathrm{~m}^{-3}$, both quantities remain almost constant, indicating a freezing effect of domain wall by space charges. It implies that the configurational force can be correlated with the energy release rate during the evolution.

Simulations have also been performed for the corresponding cases with an initial head-to-head configuration and $\alpha=60^{\circ}$. In the case without semiconducting features, the domain wall rotates to the vertical position in equilibrium. Similarly to the case of the tail-to-tail initial configuration, this is due to the driving moment. In the cases with semiconducting features, the orientation of the domain wall at the equilibrium state depends on the donor concentration. In

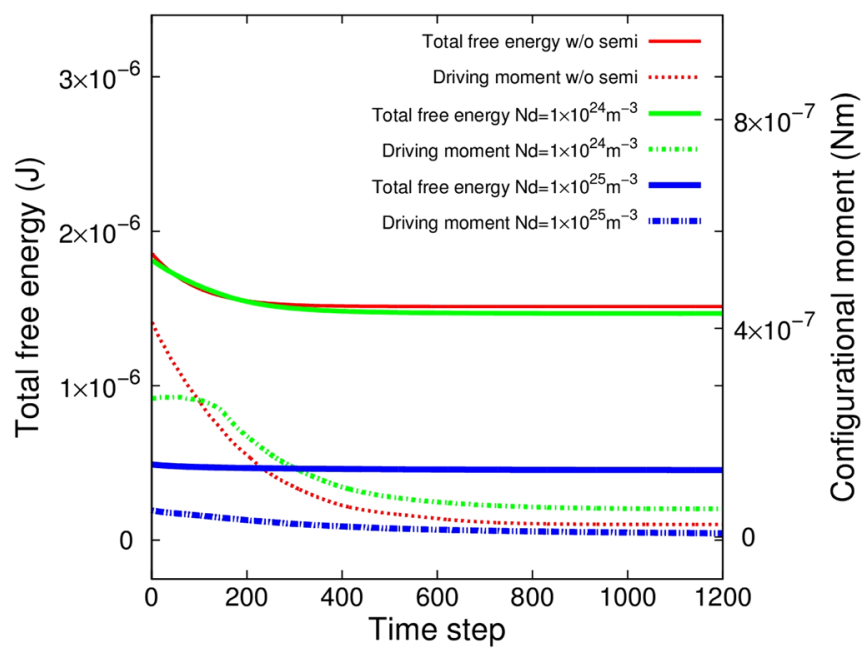

FIG. 16. Variation of the total free energy and of the configurational moment during the evolution of the domain structure into its equilibrium state. certain cases it can even evolve into a tail-to-tail structure. It should be noted that the equilibrium state can also depend on the specific choice of the Fermi level.

In this section, only the self-equilibrium of the domain configuration is considered. When an external electric field is applied, the effect of the normal driving force due to the external electric field is usually more dominant than that of the driving moment. Moreover, there is a significant anisotropy of domain wall energy. Therefore, the rotation motion of the domain wall is hardly visible. Instead, a kink-wise motion of the domain wall is observed.

The latter process, however, deals with characteristic distances at the atomic scale because domain walls in ferroelectrics have thickness of a few crystal cells as was established by electron holography. ${ }^{52,53}$ Such processes are not captured by the current phase field model based on a continuum thermodynamic approach.

\section{CONCLUSION}

In this paper, the role of donors and electronic charge carriers in the domain structure stabilization was studied in a quantitative way. By accounting for semiconducting properties of barium titanate, the appearance of depletion layers near the electrodes was predicted. The stabilization of the head-to-head and tail-to-tail domain structures through the space charge was also demonstrated.

As an indication of the stability, the driving force on the domain wall in the head-to-head domain structure was quantitatively investigated by the configurational force theory. The effects of an applied electric field and space charge compensation on the driving force and the driving moment were discussed, as well as the influence of the domain wall orientation. In particular, the results show that the considered domain wall should rotate due to the driving moment, which results from the inhomogeneous internal electric field due to the uncompensated polarization charge.

The effect of the predicted driving moment was then visualized by phase field simulations. It can be seen that the equilibrium position of the domain wall in a head-to-head or tail-to-tail domain configuration depends on the compensation situation. When semiconducting features are included, the equilibrium position is related to the position of the Fermi level and the donor/acceptor concentration. Depending on these circumstances one of the above two configurations becomes more favorable. It is interesting that both head-tohead $^{32}$ and tail-to-tail ${ }^{54}$ configurations were in fact observed experimentally revealing enhanced electrical conductivity through domain walls.

In the current study only donors were considered in the system, with the purpose of identifying their role in domain wall stabilization. This choice of the model defect system was also adopted in the studies by Xiao et al. ${ }^{29}$ and Hong et $a l .^{30}$ It should be noted that the strong compensation of the charged domain wall by the semiconducting effect requires rather high donor doping of $N_{d}=10^{25} \mathrm{~m}^{-3}$ as is seen in Fig. 14(c). Typical intrinsic concentrations of the oxygen vacancies emerging due to sintering of ferroelectric ceramics at high temperature are of the order of $10^{20} \mathrm{~m}^{-3}$ as 
evaluated by Erhart and Albe. ${ }^{41}$ Due to acceptor doping of about $1 \mathrm{~mol}$. \%, the density of oxygen vacancies can be enhanced $^{55}$ up to $N_{d}=5 \times 10^{22} \mathrm{~m}^{-3}$, the test value used in this paper. In any case, Fig. 15 exhibits a rather slow logarithmic dependence of the equilibrium rotation angle on the donor density, which means that even the effect of the intrinsic donor defects should be remarkable. However, an apparent saturation of the rotation angle at low donor densities might also mean the stabilization of the charged domain wall by electronic carriers alone. In the further study, a defect system obtained by the first principle calculations by Erhart and Albe $^{41}$ will be included in the phase field simulations. In the future work, the diffusion of the oxygen vacancies will also be incorporated, and their interaction with domain structure will be further studied. In doing so, the formulation of the configurational force should also be modified correspondingly so that the additional contribution by the diffusion into the driving force can be taken into account.

\section{ACKNOWLEDGMENTS}

The financial support by the German Science Foundation (Deutsche Forschungsgemeinschaft, Sonderforschungsbereich 595 Fatigue in Functional Materials) is gratefully acknowledged.

${ }^{1}$ Y. Watanabe, Phys. Rev. B 57, 789 (1998).

${ }^{2}$ S. B. Kim and D. Y. Kim, J. Am. Ceram. Soc. 83, 1495 (2000).

${ }^{3}$ P. V. Lambeck and G. H. Jonker, J. Phys. Chem. Solids 47, 453 (1986).

${ }^{4}$ X. Wu and D. Vanderbilt, Phys. Rev. B 73, 020103 (2006).

${ }^{5}$ M. Y. Gureev, A. K. Tagantsev, and N. Setter, Phys. Rev. B 83, 184104 (2011).

${ }^{6}$ P. Mokrý, A. K. Tagantsev, and J. Fousek, Phys. Rev. B 75, 094110 (2007).

${ }^{7}$ M. Y. Gureev, P. Mokrý, A. K. Tagantsev, and N. Setter, Phys. Rev. B 86, 104104 (2012).

${ }^{8}$ E. A. Eliseev, A. N. Morozovska, G. S. Svechnikov, V. Gopalan, and V. Y. Shur, Phys. Rev. B 83, 235313 (2011).

${ }^{9}$ M. Peach and J. S. Koehler, Phys. Rev. 80, 436 (1950).

${ }^{10} \mathrm{~J}$. D. Eshelby, in Inelastic Behavior of Solids, edited by M. F. Kanninen et al. (McGraw-Hill, New York, 1970).

${ }^{11}$ J. D. Eshelby, J. Elasticity 5, 321 (1975).

${ }^{12}$ M. E. Gurtin, Configurational Forces as Basic Concepts of Continuum Physics, Applied Mathematical Sciences Vol. 137 (Springer, New York, 2000).

${ }^{13}$ D. Gross, R. Mueller, and S. Kolling, Mech. Res. Commun. 29, 529 (2002).

${ }^{14}$ A. I. Arvanitakis, E. I. Chronaiou, and V. K. Kalpakides, Composites, Part B 43, 2628 (2012).

${ }^{15}$ B. Budiansky and J. R. Rice, J. Appl. Mech. 40, 201 (1973).

${ }^{16}$ J. W. Eischen and G. Herrmann, J. Appl. Mech. 54, 388 (1987).

${ }^{17}$ E. K. Agiasofitou and V. K. Kalpakides, Int. J. Eng. Sci. 44, 127 (2006).

${ }^{18}$ B. X. Xu, D. Schrade, D. Gross, and R. Mueller, Int. J. Fract. 166, 163 (2010).

${ }^{19}$ Y. L. Li, S. Y. Hu, Z. K. Liu, and L. Q. Chen, Acta Mater. 50, 395 (2002).
${ }^{20}$ J. Wang, S.-Q. Shi, L.-Q. Chen, Y. Li, and T.-Y. Zhang, Acta Mater. 52, 749 (2004).

${ }^{21}$ W. Zhang and K. Bhattacharya, Acta Mater. 53, 185 (2005).

${ }^{22}$ A. Soh, Y. Song, and Y. Ni, J. Am. Ceram. Soc. 89, 652 (2006).

${ }^{23}$ D. Schrade, R. Mueller, B. X. Xu, and D. Gross, Comput. Methods Appl. Mech. Eng. 196, 4365 (2007).

${ }^{24}$ R. Müller, D. Gross, D. Schrade, and B. X. Xu, Int. J. Fract. 147, 173 (2007).

${ }^{25}$ Y. H. Zhang, J. Y. Li, and D. N. Fang, J. Appl. Phys. 107, 034107 (2010).

${ }^{26}$ J. Wang, Y. F. Xia, L. Q. Chen, and S. Q. Shi, J. Appl. Phys. 110, 114111 (2011).

${ }^{27}$ Y. Su, H. T. Chen, J. J. Li, A. K. Soh, and G. J. Weng, J. Appl. Phys. 110, 084108 (2011).

${ }^{28}$ V. Stepkova, P. Marton, and J. Hlinka, J. Phys.: Condens. Matter 24, 212201 (2012).

${ }^{29}$ Y. Xiao, V. B. Shenoy, and K. Bhattacharya, Phys. Rev. Lett. 95, 247603 (2005).

${ }^{30}$ L. Hong, A. K. Soh, Q. G. Du, and J. Y. Li, Phys. Rev. B 77, 094104 (2008).

${ }^{31}$ T. Sluka, A. K. Tagantsev, D. Damjanovic, M. Gureev, and N. Setter, Nat. Commun. 3, 748 (2012).

${ }^{32}$ T. Sluka, A. K. Tagantsev, P. Bednyakov, and N. Setter, Nat. Commun. 4, 1808 (2013)

${ }^{33}$ L. Q. Chen, J. Am. Ceram. Soc. 91, 1835 (2008).

${ }^{34}$ M. Kamlah, Continuum Mech. Thermodyn. 13, 219 (2001).

${ }^{35}$ B. X. Xu, D. Schrade, R. Müller, D. Gross, T. Granzow, and J. Rödel, Z. Angew. Math. Mech. 90, 623 (2010).

${ }^{36}$ L. D. Landau and E. M. Lifshitz, Electrodynamics of Continuous Media (Pergamon Press, New York, 1984).

${ }^{37}$ D. J. Franzbach, B. X. Xu, R. Mueller, and K. G. Webber, Appl. Phys. Lett. 99, 162903 (2011)

${ }^{38}$ D. Schrade, B. X. Xu, R. Mueller, and D. Gross, in Proceedings of the ASME 2008 Smart Materials, Adaptive Structures and Intelligent Systems (SMASIS2008) (Ellicot City, MD, 2008), p. 301.

${ }^{39}$ S. M. Sze and K. K. Ng, Physics of Semiconductor Devices (Wiley, 2007).

${ }^{40}$ P. van Halen and D. L. Pulfrey, J. Appl. Phys. 57, 5271 (1985).

${ }^{41}$ P. Erhart and K. Albe, J. Appl. Phys. 104, 044315 (2008).

${ }^{42}$ Different values have been used for $N_{d}$ in specific examples.

${ }^{43}$ Y. A. Genenko, O. Hirsch, and P. Erhart, in 2012 International Symposium on Applications of Ferroelectrics Held Jointly with 11th IEEE ECAPD and IEEE PFM (ISAF/ECAPD/PFM), IEEE International Symposium on Applications of Ferroelectrics (Aveiro, Portugal, 2012).

${ }^{44}$ J. Wang and M. Kamlah, Appl. Phys. Lett. 93, 042906 (2008).

${ }^{45}$ C.-L. Jia, S.-B. Mi, K. Urban, I. Vrejoiu, M. Alexe, and D. Hesse, Nat. Mater. 7, 57 (2008).

${ }^{46}$ G. A. Maugin, Material Inhomogeneities in Elasticity (Chapman \& Hall/CRC, 1993).

${ }^{47}$ R. Mueller and G. A. Maugin, Comput. Mech. 29, 52 (2002).

${ }^{48}$ R. Mueller, S. Kolling, and D. Gross, Int. J. Numer. Methods Eng. 53, 1557 (2002).

${ }^{49}$ Y. A. Genenko and D. C. Lupascu, Phys. Rev. B 75, 184107 (2007).

${ }^{50}$ Y. A. Genenko, Phys. Rev. B 78, 214103 (2008).

${ }^{51}$ M. B. Okatan and S. P. Alpay, Appl. Phys. Lett. 95, 092902 (2009).

${ }^{52}$ X. Zhang, T. Hashimoto, and D. C. Joy, Appl. Phys. Lett. 60, 784 (1992).

${ }^{53}$ X. Zhang, D. C. Joy, Y. Zhang, T. Hashimoto, L. Allard, and T. A. Nolan, Ultramicroscopy 51, 21 (1993).

${ }^{54}$ D. Meier, J. Seidel, A. Cano, K. Delaney, Y. Kumagai, M. Mostovoy, N. A. Spaldin, R. Ramesh, and M. Fiebig, Nat. Mater. 11, 284 (2012).

${ }^{55}$ Y. A. Genenko, O. Hirsch, and P. Erhart, Surface potential at a ferroelectric grain due to asymmetric screening of depolarization fields, arXiv:1402.2802.

This article may be downloaded for personal use only. Any other use requires prior permission of the author and AIP Publishing. This article appeared in Journal of Applied Physics 115, 084110 (2014) and may be found at https://doi.org/10.1063/1.4866359.

Available under only the rights of use according to UrhG. 